\title{
Indagine epidemiologica relativa alla prevenzione e terapia delle alterazioni del metabolismo calcio-fosforo nel paziente uremico in trattamento emodialitico in Toscana
}

\author{
S. Bandini ${ }^{1}$ e M. Lombardi ${ }^{2}$, per i Referenti dei Centri Dialisi della Toscana ${ }^{*}$ \\ ${ }^{1}$ Nefrologia dei Trapianti e Dialisi, Azienda Ospedaliera Universitaria Careggi, Firenze \\ ${ }^{2}$ U.O. Nefrologia e Dialisi, Ospedale S.M. Annunziata, Azienda Asl di Firenze
}

\section{Introduzione}

Numerosi studi multicentrici effettuati su ampie casistiche (COSMOS, DOPPS) hanno attestato che i risultati ottenuti con la terapia tradizionale (chelanti a base di calcio, alluminio, calcitriolo per os o endovena) sono stati assai deludenti. Ancora di più, ed è quello che per i nefrologi in prima linea conta, l'esperienza personale ha confermato la notevole difficoltà a raggiungere e mantenere nel tempo i livelli ematici dei principali parametri del metabolismo calcio-fosforo nel range previsto dalle Linee Guida K-DOQI (Fig. 1) (1-5). Per quanto riguarda gli anni passati, i dati eccessivamente ottimistici della letteratura dei primi anni Novanta sulla efficacia del calcitriolo ev, preconizzato come capace di determinare nel trattamento dell'iperparatiroidismo (IPT) severo una "paratiroidectomia farmacologica", non sono stati spesso confermati dalla pratica clinica quotidiana probabilmente anche per un inadeguato utilizzo di tale vitamina da parte del nefrologo. Negli ultimi anni sono divenuti però disponibili nuovi farmaci: chelanti del fosforo non contenenti né calcio, né alluminio (sevelamer e carbonato di lantanio), analoghi meno ipercalcemizzanti della vitamina D e novità

* Referenti Centro Dialisi della Toscana, si veda pag. 28. assoluta per specificità di azione e potenza, i calciomimetici (9-14). I dati della letteratura attualmente a nostra disposizione sull'uso di tali presidi terapeutici sembrano essere assai promettenti in termini di efficacia e tollerabilità; esistono però ancora molti dubbi sui migliori approcci terapeutici nel trattamento dell'osteopatia renale, in parte riferibili a una non adeguata conoscenza sui potenziali effetti negativi a lungo termine di tali farmaci (carbonato di lantanio e calciomimetici) $(15,16)$, in parte alla carenza attuale in questo settore delle linee guida i cui suggerimenti si basano assai poco su evidenze e molto più su opinioni personali (17). Questi presupposti e la nostra personale esperienza clinica ci hanno indotti ad attivare una in-

\begin{tabular}{|l|l|}
\hline Parameter & Target range \\
\hline iPTH & $\begin{array}{l}150-300 \mathrm{pg} / \mathrm{mL} \\
{[16.5-33.0 \mathrm{pmol} / \mathrm{L}]}\end{array}$ \\
\hline Ca $\times \mathrm{P}$ & $\begin{array}{l}<55 \mathrm{mg}^{2} / \mathrm{dL}^{2} \\
{\left[<4.51 \mathrm{mmol} / \mathrm{L}^{2}\right]}\end{array}$ \\
\hline Phosphorus & $\begin{array}{l}3.5-5.5 \mathrm{mg} / \mathrm{dL} \\
{[1.10-1.78 \mathrm{mmol} / \mathrm{L}]}\end{array}$ \\
\hline 'Corrected' calcium & $\begin{array}{l}8.4-9.5 \mathrm{mg} / \mathrm{dL} \\
{[2.10-2.37 \mathrm{mmol} / \mathrm{L}]}\end{array}$ \\
\hline
\end{tabular}

Fig. 1 - NKF-K/DOQI bone metabolism guidelines. 
dagine epidemiologica nella nostra regione, sui dati fondamentali del metabolismo minerale e sui provvedimenti terapeutici adottati nel trattamento dell'iperparatiroidismo secondario del paziente uremico.

\section{Metodi}

L'indagine è stata effettuata mediante invio di un questionario per email, posta di superficie o fax ai 32 Centri Dialisi toscani. Al fine di ottenere la maggiore adesione possibile il questionario è stato limitato a pochi $(n=10)$ e semplici quesiti. Per rendere realizzabile la raccolta dati in tempi accettabili si è fatto riferimento alla più recente determinazione ematochimica effettuata da ogni Centro in un arco temporale di 4 mesi nel 2007 che comprendesse i parametri bioumorali considerati. In particolare il questionario ha preso in esame i seguenti punti:

- Valori di fosforemia $(\mathrm{mg} / \mathrm{dL})$, prodotto CaxP $\left(\mathrm{mg}^{2} / \mathrm{dl}^{2}\right)$, paratormone intatto (iPTH) (pg/mL);

- Uso di chelanti del fosforo sia in monoterapia che in associazione;

- Approccio terapeutico preferito nel trattamento dell'IPT secondario in base al grado di severità; - Uso di vitamina D o suoi analoghi e/o calciomimetici;

- Dosi medie settimanali dei suddetti farmaci nella fase di mantenimento;

- Numero di paratiroidectomie effettuate nel 2006. Per la elaborazione statistica dei dati è stato usato il test $T$ di Student per dati appaiati.

\section{Risultati}

Hanno risposto al questionario 28 Centri Dialisi su 32. Ciò ha reso possibile l'elaborazione statistica dei dati provenienti da 1822 pazienti uremici in trattamento cronico emodialitico extracorporeo.

Le prime 3 domande del questionario erano volte a rilevare $\mathrm{i}$ dati complessivi dei principali parametri bioumorali del metabolismo minerale (fosforemia, prodotto CaxP, PTH) e a calcolare per ogni parametro la percentuale di pazienti che rientravano nel range di valori indicato come target dalle linee guida K-DOQI (Fig. 1).

La distribuzione dei pazienti in base ai livelli di fosforemia è mostrata in Tabella I.

Il 51\% dei pazienti raggiungeva il target di fosforemia previsto dalle linee guida K-DOQI. (Tab. I, Fig. 1) ma questa percentuale godeva di ampia variabilità tra i singoli Centri. Nel Centro "più virtuoso" il 70\% dei pazien- ti rientrava nel range di valori ottimali mentre in quello "meno virtuoso" tale valore si riduceva al 35\%. All'opposto, il 49\% dei pazienti presentava livelli di fosforemia fuori dal range proposto dalle linee guida (Fig. 2). Fra questi ultimi il mancato raggiungimento dei valori target era dovuto prevalentemente ad iperfosforemia (27\%) piuttosto che ad ipofosforemia (22\%), quest'ultima verosimile espressione di pazienti anziani e/o malnutriti (Tab. I , Fig. 2).

Riguardo al prodotto CaxP, il 76\% dei pazienti rientrava nel range raccomandato dalle linee guida K-DOQI (Tab. II, Fig.2) ma anche in questo caso erano presenti sostanziali differenze percentuali fra i vari Centri (26\% e 100\% rispettivamente nel Centro meno e in quello più virtuoso). Da notare comunque che circa un quarto (24\%) di tutti i pazienti (Tab.II, Fig.2) ha un valore di prodotto CaxP $>55 \mathrm{mg}^{2} / \mathrm{dL}^{2}$, ovvero a rischio significativo di calcificazioni vascolari.

I dati del terzo quesito, relativo alla distribuzione percentuale dei pazienti in base ai livelli di PTH intatto iPTH, documentavano che solo il 36\% dei pazienti rientrava nel range raccomandato $(150-300 \mathrm{pg} / \mathrm{mL})$, anche in questo caso con ampia variabilità percentuale tra Centri più e meno virtuosi ( $54 \%$ vs $16 \%$ ). Complessivamente, ben il $64 \%$ dei pazienti mostrava livelli sierici di iPTH al di fuori del range suggerito dalle linee guida

TABELLA I - DISTRIBUZIONE DEI PAZIENTI IN BASE AI LIVELLI DI FOSFOREMIA

$\begin{array}{ll}\mathrm{P}<3.5 \mathrm{mg} / \mathrm{dL} & 22.1 \% \\ \mathrm{P} \text { tra } 3.5-5.5 \mathrm{mg} / \mathrm{dL} & 51.2 \% \\ \mathrm{P}>5.5 \mathrm{mg} / \mathrm{dL} & 26.7 \%\end{array}$

TABELLA II - DISTRIBUZIONE DEI PAZIENTI IN BASE AI VALORI DI PRODOTTO CaxP

$\begin{array}{ll}\mathrm{CaxP}<55 \mathrm{mg}^{2} / \mathrm{dL}^{2} & 76 \% \\ \text { CaxP tra } 55-65 \mathrm{mg}^{2} / \mathrm{dL}^{2} & 14 \% \\ \text { CaxP }>65 \mathrm{mg}^{2} / \mathrm{dL}^{2} & 10 \%\end{array}$

TABELLA III - DISTRIBUZIONE DEI PAZIENTI IN BASE AI LIVELLI DI PTH INTATTO

iPTH $<150 \mathrm{pg} / \mathrm{ml}$

$39 \%$

iPTH tra $150-300 \mathrm{pg} / \mathrm{ml} \quad 36 \%$

iPTH tra $301-500 \mathrm{pg} / \mathrm{ml} \quad 16 \%$

$\mathrm{iPTH}>500 \mathrm{pg} / \mathrm{ml}$ 


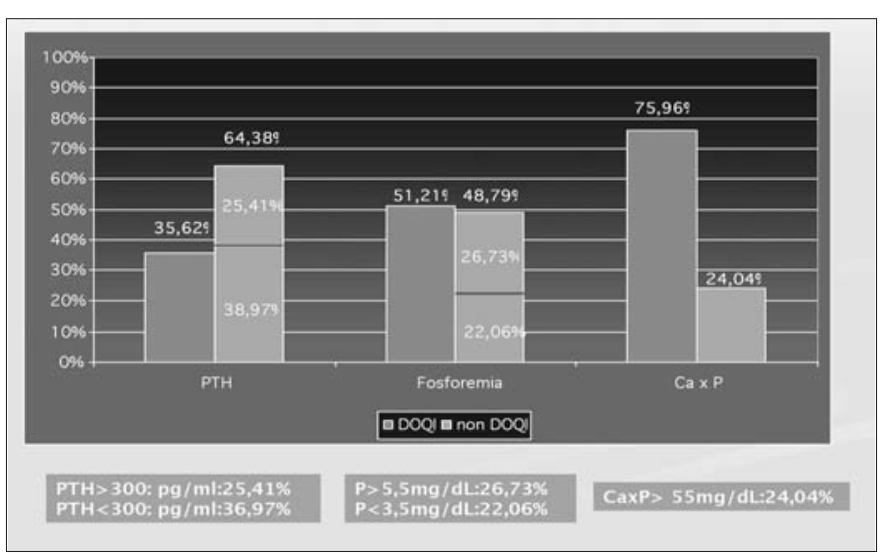

Fig. 2 - Distribuzione percentuale dei pazienti che rientrano nei parametri delle Linee Guida DOQI.

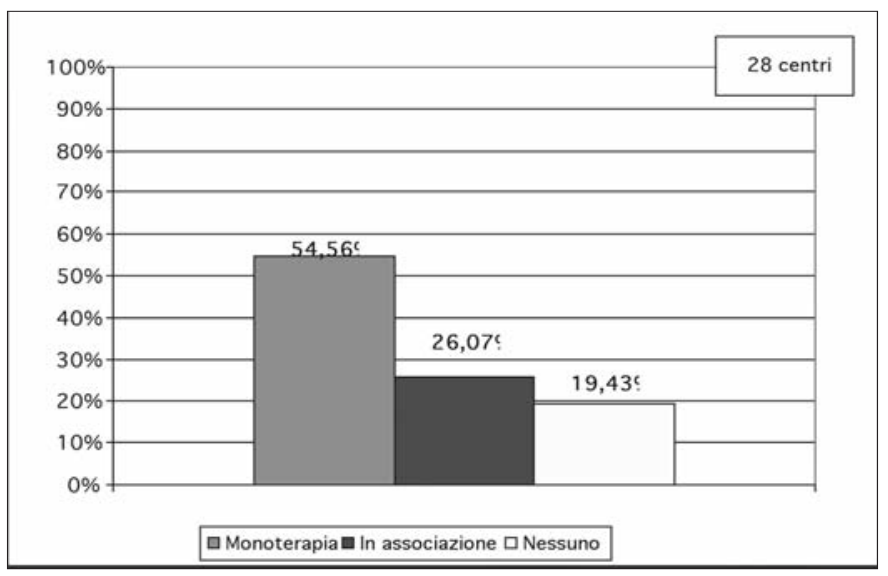

Fig. 4 - Distribuzione percentuale dei pazienti che utilizzano chelanti in monoterapia $o$ in associazione.

(Fig. 2). Inoltre, in accordo con i dati della letteratura, anche nella nostra regione si confermava la prevalenza di pazienti con bassi livelli di iPTH $(<150 \mathrm{pg} / \mathrm{mL})$ (Tab. III, Fig. 2), rilevando una maggior tendenza all'ipoparatiroidismo piuttosto che all'iperparatiroidismo.

Gli ultimi quesiti valutavano l'approccio terapeutico dei nefrologi toscani alle alterazioni del metabolismo calcio-fosforico nei pazienti in trattamento emodialitico. Il primo obiettivo è stato quello di valutare l'effettivo e attuale uso dei chelanti dei fosfati. Deve essere tenuto presente che al tempo dell'invio del questionario il carbonato di lantanio si era reso disponibile nei Centri indagati solo da pochi mesi.

L'81\% della popolazione dialitica esaminata utilizzava chelanti del fosforo (Fig. 3), preferenzialmente in monoterapia piuttosto che in associazione (55\% vs $26 \%$ ) (Fig. 4). I chelanti a base di calcio e il sevelamer erano quelli più utilizzati sia in monoterapia sia in associazione (Figg. 5-6). È comunque intrigante rilevare come in

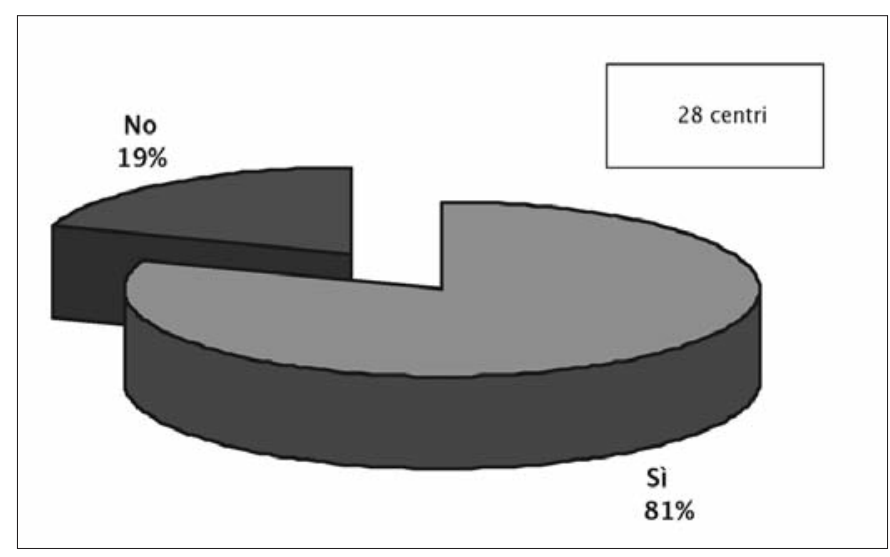

Fig. 3 - Distribuzione percentuale dei pazienti che utilizzano chelanti del $\mathbf{P}$.

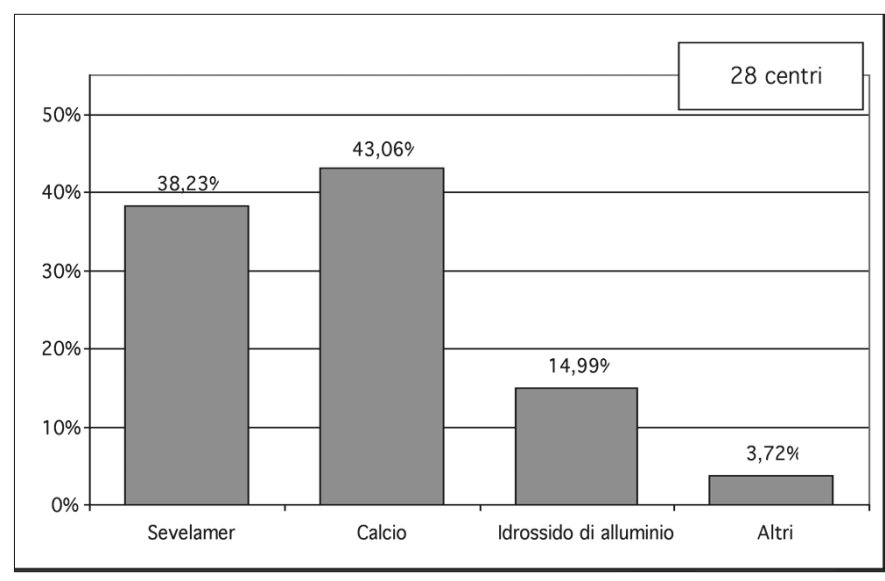

Fig. 5 - Distribuzione percentuale dei pazienti che utilizzano chelanti dei fosfati in monoterapia.

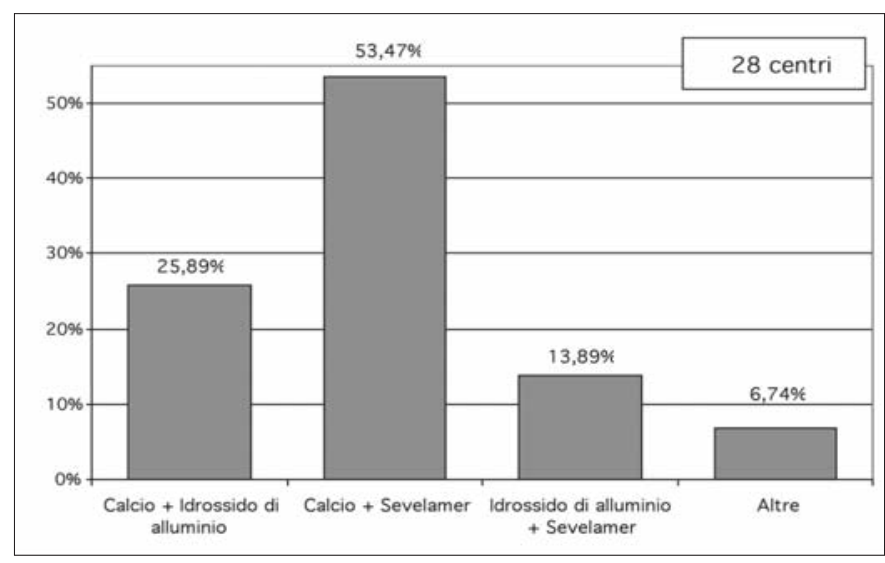

Fig. 6 - Distribuzione percentuale dei pazienti che utilizzano chelanti dei fosfati in associazione.

Toscana i chelanti a base di alluminio erano ancora utilizzati in associazione a carbonato di calcio o sevelamer nel 30\% dei pazienti. Tra i pazienti che utilizzavano 


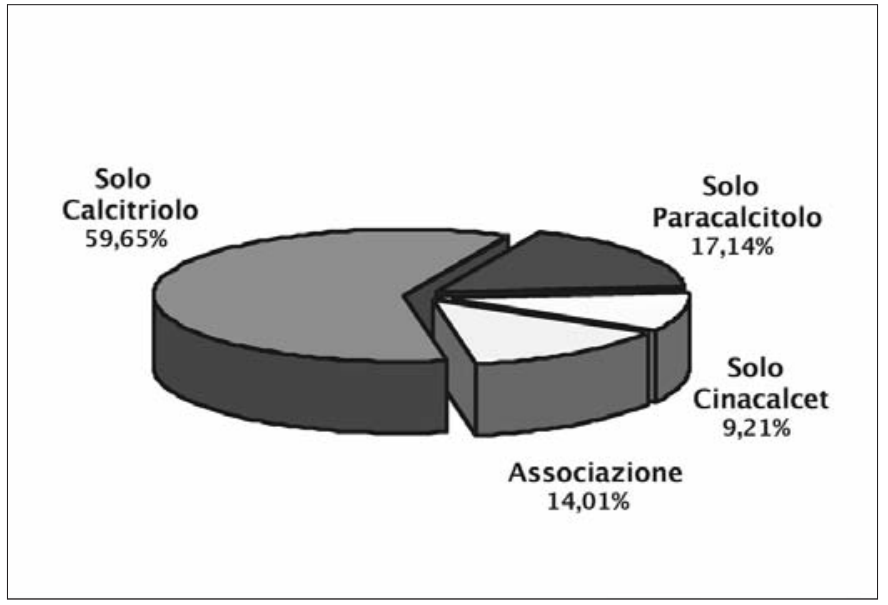

Fig. 7 - Distribuzione percentuale dei pazienti che assumono vitamina D (osev) e/o calciomimetrico.

chelanti in monoterapia ben il $15 \%$ assumeva idrossido di alluminio (Figg. 5, 6).

Il questionario prevedeva poi 4 domande relative alla distribuzione percentuale di pazienti che nei Centri esaminati, assumevano vitamina D (os-ev) o calciomimetico e all'approccio farmacologico "preferito" dai "nefrologi toscani", in presenza di segni bioumorali di IPT secondario di vario grado. Volevamo valutare se e quanto, le più recenti opzioni terapeutiche erano utilizzate comunemente dal nefrologo.

Va tenuto presente, relativamente al I quesito che il calcitriolo per os (a dosi fisiologiche), viene regolarmente somministrato ai pazienti in dialisi, a prescindere dalla presenza o meno di iperparatiroidismo: era pertanto possibile un "overlap" nelle risposte fornite. Dei 1822 pazienti esaminati, il 56\% assumeva almeno un farmaco per il trattamento dell'IPT. Il farmaco in assoluto più impiegato (in circa il 60\% dei pazienti) era il calcitriolo per os; il restante $40 \%$ dei pazienti trattati utilizzava o solo paricalcitolo o solo cinacalcet, oppure la loro associazione (Fig. 7).

Ai 3 successivi quesiti i referenti dei singoli Centri hanno risposto che in presenza di IPT lieve (iPTH:300-500 pg/mL) preferiscono somministrare il calcitriolo (os/ev) rispetto al paricalcitolo (22 Centri Dialisi/28) (Fig. 8). Per contro, in presenza di IPT moderato (iPTH: $500-800 \mathrm{pg} / \mathrm{mL}$ ) ben 20 Centri Dialisi preferiscono somministrare il paricalcitolo in monoterapia o l'associazione vitamina $\mathrm{D}$ (paricalcitolo o calcitriolo) e calciomimetici (Fig. 9). Quest'ultima associazione, è la prima opzione terapeutica nell'IPT severo (18 Centri Dialisi /28), mentre la seconda opzione è rappresentata in percentuale sovrapponibile dal calciomimetico e dal paricalcitolo in monoterapia (Fig. 10). Solo due Centri Dialisi hanno affermato di preferire il

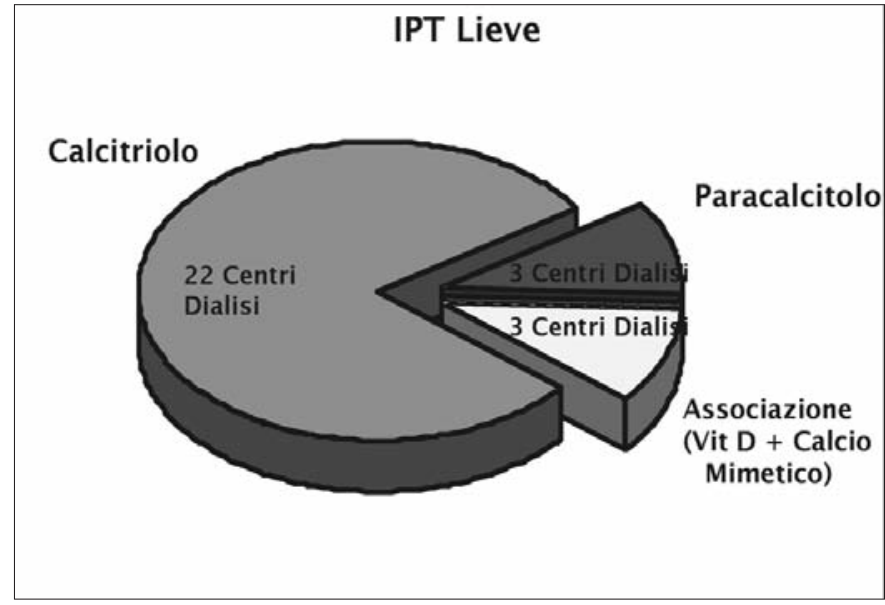

Fig. 8 - Modalità di trattamento preferito in caso di IPT lieve nei 28 Centri Dialisi.

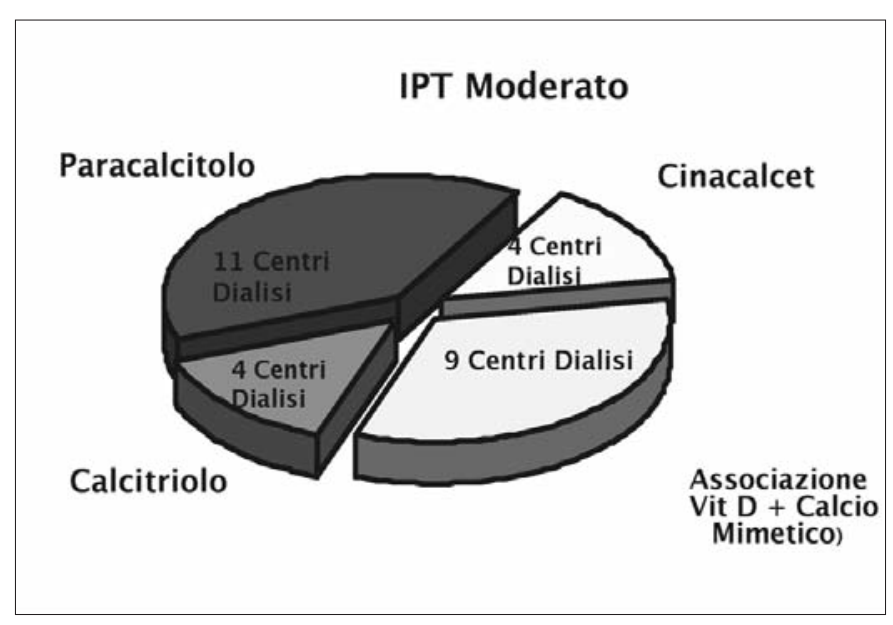

Fig. 9 - Modalità di trattamento preferito in caso di IPT moderato nei 28 Centri Dialisi.

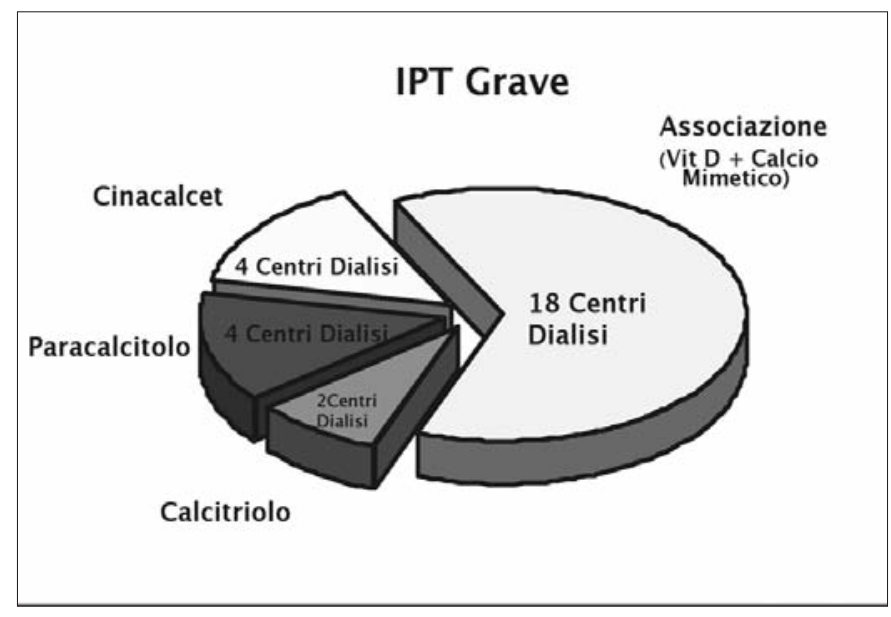

Fig. 10 - Modalità di trattamento preferito in caso di IPT severo nei 28 Centri Dialisi. 


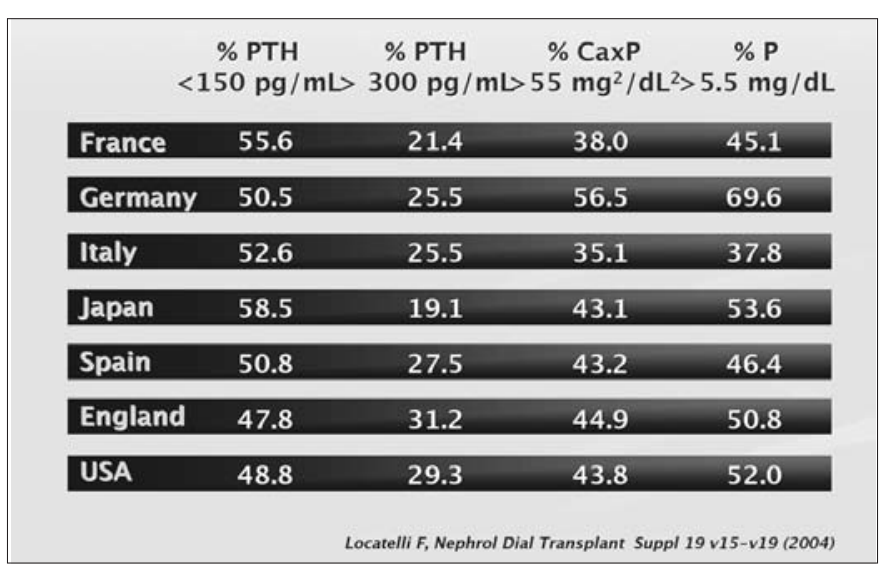

Fig. 11 - Percentage of patients within each country in the DOPPS outside the K/DOQI guideline targets.

calcitriolo in monoterapia nell'IPT severo (Fig. 10). La penultima domanda era volta alla valutazione del dosaggio medio farmacologico usato per mantenere i livelli sierici di iPTH nel range raccomandato dalle linee guida (Fig. 1).

Le dosi medie settimanali prescritte di calcitriolo e di paricalcitolo endovena non risultano significativamente diverse quando somministrati da soli o in associazione con calciomimetici (2, 7 vs $2.5 \mathrm{mcg}$ per il calcitriolo e 11.5 vs $12.7 \mathrm{mcg}$ per il paricalcitolo $\mathrm{p}=\mathrm{NS}$ ). La stessa considerazione vale per il calciomimetico, la cui dose giornaliera non differisce significativamente qualora assunto da solo o in associazione con analoghi della vitamina D (38.2 e $35.5 \mathrm{mg} / \mathrm{die} ; \mathrm{p}=\mathrm{NS})$. L'ultima domanda era volta a indagare quanto sia ancora oggi frequente il ricorso all'intervento di paratiroidectomia (PTX). Nel 2006 risultavano complessivamente 7 PTX, pari a un'incidenza di 4 PTX/1000 pazienti/anno. Può essere rilevante tuttavia osservare che tutti questi interventi furono distribuiti fra soli 4 Centri dei 28 partecipanti.

\section{Discussione}

Obiettivo della nostra indagine era fornire una fotografia dello stato attuale del metabolismo calcio/fosforico e del suo trattamento nei pazienti sottoposti ad emodialisi cronica nella nostra regione. Nonostante la semplicità dei quesiti inviati ai vari Centri, l'elaborazione dei dati ottenuti ha permesso alcune considerazioni.

> In Toscana il controllo della fosforemia e del prodotto CaxP risulta sostanzialmente soddisfacente rispetto ai dati riportati dalla letteratura (2-5). Livelli di fosforemia superiori a $5.5 \mathrm{mg} / \mathrm{dL}$ e un prodotto CaxP superiore a $55 \mathrm{mg}^{2} / \mathrm{dL}^{2}$ sono infatti presenti nel $27 \%$ e nel $24 \%$ dei pazienti, percentuali inferiori a quelle riportate nel braccio nazionale dello studio DOPPS (3), pari rispettivamente al 38\% e 35\% (Fig. 11).

> Per contro il controllo dei livelli sierici di PTH è meno soddisfacente. Il $64 \%$ dei pazienti mostra valori di PTH al di fuori dei parametri KDOQI, con una prevalenza di pazienti che presenta PTH inferiore a $150 \mathrm{pg} / \mathrm{mL}$, percentualmente superiore (39\%) (Fig. 2). Questo dato, in accordo con tutti i "bracci" nazionali (e quindi anche con quello italiano) dello studio DOPPS, significa che oggi in Toscana, come in tutta Italia e in molti altri Paesi, i pazienti uremici emodializzati sono molto più a rischio di osteopatia a basso turnover piuttosto che ad alto turnover (52.6\% vs $25.5 \%)$ (Fig. 11).

> La terapia con chelanti del fosforo è a tutt'oggi ampiamente utilizzata. La necessità di ricorrere a questo tipo di trattamento è dovuta a varie cause, fra cui un insufficiente controllo dietetico dell'apporto alimentare di fosforo e la difficoltà di riuscire a incrementare la dose dialitica, ad esempio aumentando in modo significativo la durata del trattamento extracorporeo come accade per le dialisi notturne. Quest'ultimo provvedimento sarebbe di indubbia efficacia ma è in realtà di difficile praticabilità nei Centri Dialisi per motivi sia logistico/economici che di carenza di personale.

- I chelanti del fosforo più prescritti sia in monoterapia che in associazione sono quelli a base di calcio (Figg. 5, 6), in contrasto con le raccomandazioni KDOQI che consigliano di limitarne l'uso (dose consigliata $\leq 1.5 \mathrm{gr} /$ die di Ca++ ) perché "associati" ad aumentato rischio di calcificazioni vascolari (1). La maggiore sorpresa è risultata però la frequenza con cui il nefrologo prescriveva ancora il tanto demonizzato idrossido d'alluminio. Il consumo di chelanti a base di alluminio non sembra neanche essere completamente giustificato dalla necessità di ricorrere a questo tipo di farmaci nei casi più complessi, dato che in ben il 15\% di tutta la popolazione dialitica esaminata essi sono somministrati in monoterapia (Fig. 6).

Si può pertanto affermare che non solo il chelante ideale ancora non esiste ma che a tutt'oggi i chelanti più recenti non sembrano aver ancora del tutto sostituito quelli più datati.

> La letteratura più recente ha individuato nel paricalcitolo l'ormone più selettivo (con attività ipercalcemizzante ed iperfosforemizzante 10 volte inferiore a 
quella del calcitriolo), fra quelli attualmente disponibili, nell'attivare i recettori VDR sulle ghiandole paratiroidee e ha attribuito a questa molecola effetti positivi su outcomes "pesanti" quali mortalità globale e da cause cardiovascolari (16-18). Nonostante questa "messe" di dati in favore del paricalcitolo, $\mathrm{i}$ nefrologi toscani mettono ancora al primo posto nelle preferenze di trattamento dell'IPT di lieve entità il calcitriolo (Fig. 8). Per contro, nell'IPT moderato il paricalcitolo in monoterapia diviene l'opzione terapeutica preferita (Fig. 9). Il calciomimetico sembra ormai essere divenuto farmaco insostituibile nell'osteopatia di grado elevato, rappresentando l'opzione terapeutica preferita in oltre i $2 / 3$ dei Centri esaminati.

> Il ricorso all'intervento di PTX nella nostra regione è assai contenuto, risultando nel 2006 solo 4 PTX/1000 pazienti/anno. Questo dato è certamente parziale, limitato nel tempo e ovviamente non confrontabile con quello di casistiche in cui l'incidenza di PTX è stata corretta anche per l'età dialitica $(19,20)$. Tuttavia può ugualmente significare che già nel 2006 il nefrologo prestava particolare attenzione alla prevenzione dell'osteopatia ad alto turn-over nei pazienti dializzati utilizzando le diverse opzioni terapeutiche, anche le più nuove, disponibili così da non dover ricorrere alla PTX. Questa infatti rappresenta il fallimento della terapia medica, testimoniando una scarsa compliance del paziente al trattamento o, peggio ancora, una terapia inadeguata o tardiva da parte del nefrologo.

\section{Conclusioni}

L'indagine da noi svolta, con il determinante contributo e la fattiva collaborazione della stragrande maggioranza dei Centri Dialisi della Toscana, cui va il nostro sincero ringraziamento, non ha ovviamente le pretesa di rispondere agli interrogativi che questa complessa patologia pone al nefrologo. Per perseguire un tale risultato avremmo dovuto implementare e articolare le domande sui singoli punti in discussione con il rischio di ottenere una minore adesione da parte dei Centri di Dialisi e/o un più facile "inquinamento" dei dati trasmessi. Viceversa, la semplicità del questionario inviato ha permesso di ottenere tutti i risultati in un tempo relativamente breve e di esaminare un elevato numero di pazienti, oltre 1800, in modo da fornire dati statisticamente validi.

Abbiamo la convinzione di essere riusciti a presentare un quadro realistico delle alterazioni del metabolismo osseo e minerale dei nostri pazienti emodializzati e dell'approccio terapeutico adottato nella nostra regione. $\mathrm{Ci}$ auguriamo che questa "finestra epidemiologica" possa contribuire alla conoscenza e al trattamento dell'osteopatia uremica che rappresenta un obiettivo primario nella cura dei nostri pazienti.

sandro.bandini@virgilio.it

\footnotetext{
* Referenti dei Centri Dialisi della Toscana che hanno partecipato alla indagine:
}

Giancarlo Betti, Nefrologia e Dialisi, Ospedale di Massa, ASL 1 Massa Carrara; Donella Borracelli, Nefrologia e Dialisi, Poggibonsi, Siena; Beatrice Braccagli, UOC Nefrologia, Dialisi e Trapianto, Azienda Ospedaliera Universitaria Senese; Adriano Bruci, Nefrologia e Dialisi, Ospedale del Valdarno, Azienda USL8, Arezzo; Alessandro Capitanini, SA Nefrologia, Ospedale di Pescia, ASL 3; Renato Cominotto, Nefrologia e Dialisi, Ospedale di Piombino, ASL 6 di Livorno; Marina Consegna, UO Nefrologia e Dialisi, Spedali Civili, Livorno; Adamasco Cupisti, Nefrologia e Dialisi 2 Universitaria, Pisa; Roberto Daini, Nefrologia e Dialisi di Carrara, Azienda ASL di MassaCarrara; Ennio Duranti, Sezione Dipartimentale Emodialisi, USL 8 Arezzo; Giacomina Fontana, Responsabile Dialisi di Castelnuovo Garfagnana e Barga; Francesco Paolo Ferrandello, Nefrologia e Dialisi, Ospedale Versilia, Azienda ASL di Viareggio; Marco Gallo, Centro Dialisi Ulivella, Istituto Fioren- tino di Cura e Assistenza, Firenze; Claudio Gabrielli, UO Nefrologia e Dialisi, Ospedale Misericordia, ASL 9, Grosseto; Riccardo Giusti, UO Nefrologia e Dialisi, Ospedale Campo di Marte, ASL 2, Lucca; Cristina Grimaldi, SC Nefrologia e Dialisi, Ospedale San Giovanni di Dio, ASL Firenze; Patrizio Imperiali, UO Nefrologia e Dialisi, Ospedale S. Donato, ASL 8, Arezzo; Ciro Lenti, UO Nefrologia e Dialisi, USL 11, Empoli, Pisa; Alberto Lippi, UO Nefrologia dei Trapianti e Dialisi 1, Azienda Ospedaliera Universitaria Pisana, Pisa; Giorgio Maffucci, UO Nefrologia e Dialisi, Pistoia; Milena Meriggioli, Sezione Dialisi, UO Medicina Seconda, Ospedale Lotti, Pontedera; Stefano Michelassi, UO Nefrologia e Dialisi, Ospedale SM Annunziata, ASL Firenze; Piero Paparatto, Sezione di Nefrologia e Dialisi, Presidio Ospedaliero di Cecina, Azienda USL 6, Livorno; Giuseppe Pratesi, Servizio di Dialisi, Portoferraio, Livorno; Francesco Santori, Sez. Nefrologia e Dialisi, Ospedale Valdichiana, ASL 7 di Siena; Gabriella Sibilia, Servizio Emodialisi “A. Rossi”, Ospedale S. Maria Maddalena, Volterra, ASL 5 di Pisa; Lorena Traversari, Sez. Nefrologia e Dialisi, Ospedale S. Andrea, Massa Marittima, AUSL 9, Grosseto. 


\section{Bibliografia}

1. NKF- K-DOQI clinical practice guidelines for bone metabolism and disease in chronic kidney disease. Am J Kidney Dis 2003; 44 (Suppl 3).

2. Young EW, Albert MJ, Satayatum S, et al. Predictors and consequences of altered mineral metabolism: The Dialysis Outcomes and Practice Patterns Study. Kidney Int 2005; 67: 1171-87.

3. Young EW, Akiba T, Albert JM, et al. Magnitude and impact of abnormal mineral metabolism in the Dialysis Outcomes and Practice Patterns Study (DOPPS). J Kidney Dis 2004; 44: 34-8.

4. Fernandez-Martin J, Gorriz J, Ketteler M, et al. Achievement of $\mathrm{K} / \mathrm{DOQI}$ targets in European hemodialysis patients in the COSMOS study: differences between prevalent and incident patients. Presented at: Renal Week, 8-13 November 2006; Philadelphia.

5. Cannata-Andia JB,Carrera F. The pathophysiology of secondary hyperparathyroidism and the consequences of uncontrolled mineral metabolism in chronic kidney disease: the role of COSMOS. Nephrol Dial Transplant Plus 2008;1(Suppl 1): S2-6.

6. Andress DL, Norris KC, Coburn JW, Levine B. Intravenous calcitriol in the treatment of refractory osteitis fibrosa of chronic renal failure. N Engl J Med 1989; 321: 274-9.

7. Malberti F, Surian M, Cosci P. Effect of chronic intravenous calcitriol on parathyroid function and set-point of calcium in dialysis patients with refractory secondary hyperparathyroidism. Nephrol Dial Transplant 1992; 7: 822-8.

8. Lach F, Hervas J, Cerezo S. The importance of dosing intravenous calcitriol in dialysis patients with severe hyperparathyroidism. Am J Kidney Dis 1995; 26: 845-51.

9. Sprague SM, Liach F, Amdahl M, et al. Paricalcitol versus calcitriol in the treatment of secondary hyperparathyroidism. Kidney Int 2003; 63 (4): 1483-90.

10. Mittman N, Khanna R, Chattopadhyay J, et al. Paricalcitol therapy for secondary hyperparathyroidism in pa- tients on maintenance hemodialysis previously treated with calcitriol: a single center cross-over study. Kidney Int 2006; 70 (Suppl): S64-7.

11. Coyne D, Acharya M, Qiu P, et al. Paricalcitol capsule for the teatment of secondary hyperparathyroidism in stages 3 and 4 CKD. Am J Kidney Dis 2006; 47: 263-76.

12. Messa PG, Macario F, Yaqoob M, et al. The OPTIMA Study: Assessing a new Cinacalcet (Sensipar/Mimpara) treatment algorithm for secondary hyperparathyroidism. Clin J Am Soc Nephrol 2008; 3: 36-45.

13. Frazao J, Rodriguez M. Secondary hyperparathyroidism disease stabilisation following calcimimetic therapy. Nephrol Dial Transplant 2008; 1 (Suppl 1): S12-7.

14. Choncol M, Wuthrich RP. Potential future uses of calcimimetics in patients with chronic kidney disease. Nephrol Dial Transplant 2008; 1 (Suppl 1): S36-41.

15. Mazzaferro S, Cozzolino M, Marangella M, et al. Calcimimetics, phosphate binders, vitamin D and its analogues for treating secondary hyperparathyroidism in chronic kidney disease: guideline from the Italian Society of Nephrology. G Ital Nefrol 2007; 24 (Suppl 37): S107-24.

16. Slatopolsky E, Finch J, Ritter C, et al. A new analog of calcitriol,19-Nor-1,25-(OH)2D2 suppresses parathyroid hormone secretion in uremic rats in the absence of hyperpcalcemia. Am J Kidney Dis 1995; 26: 852-60.

17. Teng M, Wolf M, Lowrie E, et al. Survival of patients undergoing hemodialysis with paracalcitol or calcitriol therapy. N Engl Med 2003; 349(5): 446-56.

18. Kalantar-Zadeh K, Kuwae N, Regidor DL, et al . Survival predictability of time-varying indicators of bone disease in maintenance hemodialysis patients. Kidney Int 2006; 70: 771-80.

19. Malberti F, Marcelli D, Conte F, et al. Parathyroidectomy in patients on renal replacement therapy: an epidemiologic study. J Am Soc Nephrol 2001; 12: 1242-8.

20. Kestenbaum B, Andress DL, Schwartz M, et al. Parathyroidectomy rates among United States dialysis patients: 1990-1999. Kidney Int 2004; 65: 282-88. 